\title{
Famine et émigration au féminin : spécificités et crise des représentations
}

Famine and female emigration: specificities and crisis of representations

\author{
Nathalie Sebbane
}

\section{(2) OpenEdition}

Journals

Édition électronique

URL : http://journals.openedition.org/rfcb/273

DOI : $10.4000 /$ rfcb. 273

ISSN : 2429-4373

Éditeur

CRECIB - Centre de recherche et d'études en civilisation britannique

Édition imprimée

Date de publication : 1 septembre 2014

Pagination : 149-156

ISSN : 0248-9015

\section{Référence électronique}

Nathalie Sebbane, «Famine et émigration au féminin : spécificités et crise des représentations »,

Revue Française de Civilisation Britannique [En ligne], XIX-2 | 2014, mis en ligne le 01 mai 2015, consulté le 01 mai 2019. URL : http://journals.openedition.org/rfcb/273; DOI : 10.4000/rfcb.273

Ce document a été généré automatiquement le 1 mai 2019.

\section{(c) $($ ) $\odot \odot$}

Revue française de civilisation britannique est mis à disposition selon les termes de la licence Creative Commons Attribution - Pas d'Utilisation Commerciale - Pas de Modification 4.0 International. 


\title{
Famine et émigration au féminin : spécificités et crise des représentations
}

\author{
Famine and female emigration: specificities and crisis of representations
}

\author{
Nathalie Sebbane
}

1 Cet article propose une lecture de la Famine et de ses conséquences à travers le prisme de la spécificité de l'émigration féminine. Si les conséquences démographiques de cet épisode tragique de l'histoire irlandaise ont été largement débattues et analysées par les historiens, tant sur le plan de la mortalité que sur celui de l'émigration, la prise en compte de la dimension « genrée » de l'émigration n'a pas véritablement fait l'objet d'une étude approfondie dans l'historiographie contemporaine. Pourtant, elle permet à la fois d'appréhender la Famine sous un angle différent, plus complet et inclusif, mais offre également un point de comparaison avec les autres mouvements migratoires européens, surtout en direction des États-Unis. À la lumière de sources jusqu'ici peu explorées, cet article démontrera que l'émigration féminine fut essentiellement une émigration de femmes seules, souvent de mères célibataires, qui, en dépit des perceptions et représentations qui en étaient données, utilisèrent la migration comme un outil de librechoix, à une époque où elles étaient victimes de la plus grande stigmatisation. L'indicible horreur de la Famine pourra ainsi être dite grâce à une inclusion de la dimension spécifiquement féminine des conséquences de cet épisode.

\section{Une histoire de l'émigration écrite au masculin}

Dans les années 1980, avec le développement de la recherche en histoire des femmes, plusieurs historiennes ont consacré leurs travaux à l'étude de l'émigration féminine en Irlande. Elles ont tenté de dégager les spécificités du mouvement migratoire féminin, le plaçant dans une logique différente de l'émigration masculine. L'idée était de démonter que, si les conditions de pauvreté en Irlande, aussi bien avant que pendant et après la 
Famine, avaient été déterminantes dans les décisions des femmes de partir vers une vie meilleure, les circonstances et les modalités étaient différentes. Jusque-là, l'histoire de l'émigration avait été exclusivement écrite au masculin. Pourtant, dès 1983, Robert Kennedy dans son article révolutionnaire, The Irish: Emigration, Marriage and Fertility ${ }^{1}$ révélait que les femmes avaient en réalité dominé le mouvement migratoire vers l'extérieur du pays pendant la majeure partie du dix-neuvième et du vingtième siècle, et que les raisons qui les avaient poussées à partir ne reflétaient pas nécessairement celles des hommes. Gerda Lerner, dont les recherches font oeuvre pionnière dans les nouvelles approches méthodologiques en histoire des femmes et du genre, a insisté sur l'importance d'intégrer la dimension du genre dans tout type d'écriture de l'histoire: " Lorsque le genre est envisagé avec la race, la classe, l'appartenance ethnique et l'affiliation religieuse pour analyser une période ou un événement donné, une dimension entièrement nouvelle est ajoutée à l'histoire sociale $"^{2}$. Prendre en compte la notion de genre et repenser l'épisode de la Famine et du mouvement migratoire qu'elle a engendré du point de vue des femmes permet de lire cet épisode sous un nouvel angle, lui donnant enfin tout le sens qu'il mérite. Il s'agit de rétablir une forme de vérité historique en réintégrant au sein de l'histoire sociale du pays des éléments qui ont été occultés. Pourtant pendant longtemps, cette démarche inclusive a été ignorée.

Depuis, des historiennes ont consacré des articles ou ouvrages à l'expérience spécifique des femmes dans la grande tradition migratoire des Irlandais, notamment aux dixneuvième et vingtième siècles. Hasia Diner ${ }^{3}$ et Janet Nolan ${ }^{4}$ se sont particulièrement intéressées aux expériences des Irlandaises aux États-Unis. Diner a largement utilisé les archives américaines pour documenter sa recherche, et son ouvrage a le grand mérite de fournir un vaste échantillon de chiffres et statistiques qui, s'ils ne suffisent pas à eux seuls à expliquer le phénomène, permettent d'avoir des points de comparaison avec d'autres pays et d'analyser l'évolution du phénomène.

\section{Spécificités de l'émigration des femmes}

4 Hasia Diner qui a publié le fruit de ses recherches sur l'émigration des Irlandaises vers les États-Unis, ${ }^{5}$ confirme ce que Robert Kennedy avait avancé dans les années 1980, à savoir que les femmes représentaient la majorité des émigrants irlandais et qu'aucun aucun autre groupe émigrant vers les États-Unis à cette époque ne comprenait autant de femmes. À titre d'exemple, à la même époque, la population féminine arrivant d'Allemagne ne représentait que $41 \%$ de la population totale des émigrants allemands, alors que ce chiffre était de 52,9\% pour les Irlandaises ${ }^{6}$. Le contraste s'accentua à la fin du dix-neuvième et au début du vingtième siècle, avec des pourcentages de population féminine de plus en plus faibles parmi les émigrants allemands. Les Juifs furent les seuls dont la population féminine fut à peu près équivalente à la population masculine, si ce n'est que l'émigration juive vers les États-Unis fut essentiellement une émigration familiale, alors que l'émigration irlandaise fut en grande majorité une émigration de célibataires, aussi bien pour les hommes que pour les femmes ${ }^{7}$. Néanmoins, pour Diner, comme pour d'autres historiens de l'émigration, ce fut la Famine, ou plus précisément les conséquences de ce dramatique épisode de l'histoire du pays, tant en termes démographiques qu'en termes économiques, qui fut responsable de ce schéma spécifique en Irlande : 
Ainsi, le pourcentage et le nombre absolu de femmes immigrant depuis l'Irlande augmentèrent en proportion directe avec le durcissement des conditions familiales et des perspectives d'avenir de plus en plus limitées pour les femmes en Irlande. Les femmes ne prédominèrent pas parmi les nouveaux venus en Grande-Bretagne jusque dans les années 1890, alors que parmi les Irlandais qui choisirent de tenter leur chance en Australie, hommes et femmes arrivèrent en nombre égal jusqu'en 1911 , après quoi les femmes dominèrent en nombre. ${ }^{8}$

Différents programmes d'émigration assistée existaient en Irlande au dix-neuvième siècle. Les Gardiens des Pauvres ${ }^{9}$ pouvaient décider de financer tout ou partie du voyage des pauvres de leur circonscription, et les propriétaires terriens, eux aussi, avaient souvent intérêt à aider leurs employés à émigrer, afin de préserver leur terre et de pouvoir en convertir les cultures. Enfin, certains prisonniers étaient libérés sur des critères de bonne conduite, à condition qu'ils quittent l'Irlande.

6 Néanmoins certains programmes s'adressaient particulièrement aux femmes. Il s'agissait en premier lieu des programmes d'émigration assistée depuis la workhouse. Hasia Diner indique qu'en 1850, 2847 femmes quittèrent la workhouse pour l'Amérique. Ces départs assistés ne représentèrent, en réalité, qu'une infime partie du mouvement migratoire des femmes. En effet, dans la plupart des cas, c'est parce qu'elles y étaient encouragées par d'autres femmes, à la fois psychologiquement et financièrement, que les Irlandaises se lancèrent dans l'aventure. Comme nous l'avons déjà mentionné, il s'agissait dans la majorité des cas de célibataires, mais de nombreuses veuves, accompagnées de leurs enfants quittèrent également le pays pour aller rejoindre d'autres membres de leur famille aux États-Unis ou ailleurs. Diner ne fait aucune mention des mères célibataires, mais il est vraisemblable que parmi les femmes qui quittèrent la workhouse dans le cadre des programmes d'émigration assistés, un certain nombre était dans cette situation. Néanmoins, il est également fort probable qu'elles aient dû, afin d'accéder à ces programmes mentir sur leur statut, sachant que ces programmes ne s'adressaient qu'à des femmes à la réputation et au comportement irréprochables, mais également capables de trouver un emploi sur place. À titre d'exemple, Helen Burke ${ }^{10}$ cite un courrier expédié aux autorités de la Loi sur les Pauvres, en provenance du Canada, dans lequel il était clairement stipulé que la colonie n'accepterait des femmes que sous certaines conditions. La préférence était accordée aux jeunes femmes célibataires en bonne santé. La présence des mères célibataires était donc, de facto, exclue, sauf dans le cas où elles pouvaient laisser leurs enfants dans la workhouse et prétendre qu'elles étaient célibataires. Entre 1852 et 1872, pas moins de 15088 femmes bénéficièrent de ces programmes d'émigration assistée contre seulement 3186 hommes et 10729 enfants.

7 La difficulté majeure que pouvait rencontrer une mère célibataire qui souhaitait émigrer était d'ordre financier. Il est difficilement imaginable que la mère d'un ou plusieurs enfants illégitimes ait eu à sa disposition la somme d'argent nécessaire pour se rendre aux États-Unis, sauf si l'argent lui était envoyé par quelque parent qui y était déjà installé. En revanche, il est plus probable que l'Angleterre ait été une destination privilégiée.

8 Ann Rossiter ${ }^{11}$ insiste sur la particularité que représenta l'émigration des femmes seules : «L'étendue de cette émigration d'Irlandaises célibataires, voyageant seules, sans mari ni parents, fut une anomalie dans l'histoire générale de l'émigration européenne vers le continent américain $»^{12}$. Elle revient sur les incidences des changements structuraux de l'économie du pays pour expliquer les bouleversements auxquels les femmes devaient faire face. C'est en quête d'une place dans une autre société qu'un grand nombre de femmes quittèrent l'Irlande. Rossiter explique qu'elles entrèrent sur le marché du travail 
au plus bas de l'échelle, puisqu'elles étaient en général employées comme domestiques, couturières, ou étaient employées à l'usine.

S'il est possible de parvenir à certaines conclusions sur l'émigration des femmes seules, qu'elles soient célibataires ou filles mères, pendant et après l'épisode de la Famine, il n'en demeure pas moins que les travaux sur la question restent minces et que peu d'entre eux nous permettent d'accéder à la question des représentations. Les sources utilisées par Grace Neville, que nous allons étudier à présent, nous en offrent la possibilité.

\section{Une autre représentation de l'émigration des femmes: la contribution de la Commission du Folklore Irlandais (UCD)}

Grace Neville ${ }^{13}$ a choisi d'aborder la question de l'émigration des Irlandaises à partir de sources, à ce jour, peu exploitées par les historiens et les historiennes : les archives de la Commission du Folklore Irlandais. ${ }^{14}$ Elle regrette que les « femmes qui ont émigré, en dépit de leur grand nombre, ne soient que des ombres vacillantes à la lisière de la perception $»^{15}$. Neville explique que jusque-là, les archives de cette commission étaient généralement reconnues pour leur contenu mythologique, mais qu'une prise de conscience s'est amorcée quant à leur valeur historique, notamment en histoire des femmes. Pourtant, nous dit-elle, «cette compilation remarquable n'a été utilisée par aucune historienne de l'émigration féminine $»^{16}$. Elle fait notamment référence aux historiennes que nous avons précédemment citées. Neville reprend les grands thèmes de ce mouvement migratoire et la spécificité de l'émigration de femmes jeunes, seules et célibataires. Ce qui, dans son approche, est très intéressant, c'est que par la nature même des sources qu'elle a utilisées, nous touchons au plus près au domaine des représentations et des perceptions. Niall o'Ciosain dans un article sur l'usage de la Commission du Folklore pour l'interprétation de la Famine propose une taxinomie tripartite de la mémoire collective. Il identifie la mémoire globale, populaire et locale. La mémoire globale se situe au niveau de l'information et de l'interprétation de sources, le plus souvent écrites ; la mémoire locale est un ensemble d'histoires, de particularismes ; et la mémoire populaire consiste en

un répertoire populaire stylisé d'images, de motifs, de petits récits et de légendes surnaturelles, dont beaucoup appartiennent à un répertoire international plus large, qui forme un système de représentation de la famine et de la pénurie ainsi qu'un guide de conduite pendant de telles crises. ${ }^{17}$

Les trois niveaux de lecture peuvent se combiner :

Alors que les souvenirs de la famine étaient transmis, c'était probablement ces éléments de la mémoire locale qui correspondaient le plus à la mémoire populaire qui étaient les plus « racontables », et qui survivent dans le répertoire narratif des informateurs des années 1930 et $1940 .^{18}$

11 Les archives de la Commission du Folklore utilisées par Neville offrent donc une perspective intéressante du regard des informateurs, essentiellement des hommes assez âgés, sur les femmes qui quittèrent le pays au dix-neuvième siècle. Elle est en réalité la seule à aborder le cas de l'émigration des mères d'enfants illégitimes. Elle explique que, selon les informateurs, les femmes enceintes se voyaient refuser l'entrée sur le territoire américain, car on considérait que l'enfant serait un fardeau pour l'État. Quant au statut de mère célibataire, cela constituait un handicap majeur pour un projet d'émigration, même si l'enfant était mort. La réputation de ladite femme en faisait une persona non grata. 
Neville cite un informateur qui raconta que « les filles dont on soupçonnait qu'elles avaient été enceintes n'avaient pas le droit d'entrer aux États-Unis $»^{19}$. Selon le même informateur, les officiers de l'immigration aux États-Unis avaient un moyen infaillible de déterminer si une fille était enceinte: ils la faisaient se tenir debout sur de la glace, et pouvaient déterminer si elle attendait un enfant. Neville ne manque pas de s'interroger sur la nature de ce témoignage. Elle se demande, à juste titre, s'il s'agissait de légendes supposées faire peur aux jeunes filles qui seraient tentées de pécher en pensant qu'elles pourraient résoudre leur problème en émigrant. Cela nous renvoie au questionnement de Niall o'Ciosain sur la mémoire. Il peut s'agir de croyances populaires qui, aux confins de la mémoire locale et de la mémoire populaire, deviennent les axes d'un récit historique sur une période donnée et dans un contexte donné. Le principe de ces récits était de véhiculer la notion de péché et d'inévitabilité de la punition, quelle que fût la forme sous laquelle elle s'exprimait.

Quoi qu'il en soit, la décision d'émigrer pouvait être motivée par différentes circonstances. Il pouvait s'agir d'une jeune femme enceinte d'un homme qui acceptait de l'épouser, mais uniquement aux États-Unis et non en Irlande, pour échapper à la honte. Ou bien, les femmes étaient mères d'un enfant illégitime et émigraient sous la pression de leur famille ou de la société. Mais, ce que Grace Neville voit dans ces récits, c'est une perception des femmes comme « créatures unidimensionnelles qui, contrairement aux hommes, décidaient d'émigrer à cause d'un aspect de leur relation avec l'autre sexe ${ }^{20}$. Or, les statistiques prouvent que c'était loin d'être le cas, puisque la plus grande partie des femmes qui émigrèrent à cette époque le firent seules, alors qu'elles étaient célibataires et sans attaches. Cette dimension de choix, de libre-arbitre, ne semble pas s'être inscrite dans la mémoire des informateurs et de ce fait, échappe à l'histoire de l'émigration des femmes.

On sait finalement très peu de choses sur l'émigration des mères célibataires pendant et juste après la Famine, et certaines informations semblent contradictoires. On peut néanmoins circonscrire un certain nombre de traits caractéristiques. Une grossesse prénuptiale était considérée comme un facteur déstabilisant dans une société en perdition. Les difficultés pour un père de trouver un époux à sa fille, si celle-ci était vierge, devenaient une impossibilité si elle était enceinte. Plus que jamais, pendant et juste après la Famine, les considérations morales et religieuses furent largement dépassées par des considérations purement économiques, voire de survie. Ainsi, la possibilité pour la famille de voir disparaitre ce fardeau était inespérée. L'émigration devenait un moyen de se débarrasser d'une bouche à nourrir et d'échapper aux stigmates attachés à l'illégitimité. Pour la femme, en revanche, il est probable que les choses ne furent pas si simples. Le départ forcé et la rupture avec la famille, les amis et l'environnement, s'ajoutaient à la crainte de l'inconnu. Il est certain qu'un grand nombre de ces femmes durent abandonner leur enfant pour pouvoir partir. Le nombre d'enfants abandonnés dans les workhouses à cette époque semble le confirmer. En 1844, 22585 enfants étaient résidents des différentes workhouses du pays. À la fin du mois de février 1847, ils étaient $63000^{21}$. Le nombre d'enfants augmenta par rapport à celui des adultes car de nombreux enfants survécurent à leurs parents. Certaines workhouses étaient largement dépassées par le nombre d'enfants à leur charge. En juin 1849, il y avait 90289 enfants dans les workhouses d'Irlande. Certains enfants étaient définitivement abandonnés, d'autres l'étaient de manière temporaire, le temps pour les parents de trouver de quoi survivre. D'autres, enfin, étaient laissés aux soins de la workhouse le temps que les parents émigrent et qu'ils envoient ensuite l'argent pour payer la traversée de 
leurs enfants. Robins ${ }^{22}$ indique qu'à partir de 1847 les références aux enfants abandonnés dans les procès-verbaux des workhouses devinrent fréquentes ${ }^{23}$.

La question de la ou des représentations de la Famine a été soulevée par de nombreux écrivains et historiens. Au-delà de cette question, la possibilité même de représenter un tel épisode s'est posée. À l'instar de grandes tragédies comme l'Holocauste, la Famine a rencontré une véritable crise des représentations, tant il est difficile, voire impossible de nommer l'innommable ou de dire l'indicible. Steiner, en référence à l'Holocauste, avait parlé de l'« échec du mot »"24. L'écrivain irlandais John Banville qualifie la représentation de la Famine d'« inexprimable exprimé » ${ }^{25}$. Il n'est bien entendu pas question d'assimiler les deux événements. En revanche, les questions relatives aux représentations sont pertinentes dans les deux cas. Dans son ouvrage, The Feminization of Famine ${ }^{26}$, Margaret Kelleher explique que la tragédie de la Famine a été enveloppée de silence, tant sur le plan historiographique que dans la mémoire populaire, et ce, jusqu'à très récemment. Silence ou refoulement? Nécessité d'oublier pour aller de l'avant? Pour Kelleher, «on peut raconter la tragédie de la Famine comme une histoire de résistance ou de victimisation passive ${ }^{27}$. Elle considère cependant que l'aspect le plus caractéristique du texte littéraire sur la Famine est son potentiel à individualiser la crise. Cette considération est d'autant plus pertinente dans la mesure où, "dans la majorité des représentations de la Famine, l'individu isole", la victime de la Famine qui fait l'objet d'une description détaillée, est une femme ${ }^{28}$. En cas de crise des représentations, l'indicible parvient à être dit par le biais de représentations féminines. Dans le contexte de la Famine, l'image de la Mère, la nourricière, ne pouvant plus nourrir ses enfants, véhicule un fort pathos. La plupart des descriptions de scènes relatives à cet épisode, surtout au dix-neuvième siècle, contenaient un élément féminin, maternel surtout. Kelleher cite le roman de William Carleton, The Black Prophet, dans lequel un personnage, Margaret Murtagh, est une mère célibataire. Elle meurt dans le chapitre six du roman, et le narrateur fait le commentaire suivant: " C'était vrai : la malheureuse était passée de vie à trépas; mais on ne sut jamais si ce fut parce que son cour était brisé par le péché, la honte et l'abandon, ou à cause de la Famine et de la pression exercée par le désarroi de la misère $»^{29}$. Pour Kelleher, la première possibilité renvoie à une représentation conventionnelle de la mère célibataire confrontée aux conséquences de son péché, et annule toute inclusion de cette femme dans la sphère de la victimisation politique. "À cause du "péché" lié à son statut de mère célibataire, la vulnérabilité de Margaret et la victimisation sont d'une nature morale plutôt que socio-économique, oblitérant davantage les détails de sa famine $»^{30}$. Le statut de la mère célibataire, en période de crise, est celui d'une femme dont le péché et ses conséquences la rendent d'autant plus vulnérable. De même, les représentations féminines en général, dans le contexte de la Famine, insistent davantage sur la dimension maternelle et l'échec de la Mère à sauver l'enfant, à accomplir son rôle de protectrice.

15 Ainsi, il apparaît qu'une lecture et analyse des perceptions et représentations de l'émigration des femmes, et plus particulièrement des femmes seules, dans le contexte de la Famine, permet de dépasser la dichotomie traditionnelle idéologique de l'interprétation de cet épisode. En utilisant une grille de lecture « genrée » de la Famine, et des sources nouvelles et peu exploitées dans l'historiographie nationale, il est possible de proposer une interprétation différente et ouvrir la voie à un champ de recherche potentiel plus vaste. 


\section{NOTES}

1. Robert KENNEDY, "The Irish: Emigration, Marriage and Fertility ", Journal of Marriage and the Family, Vol. 36, No. 4, nov. 1974, pp. 832-834.

2. "When gender is considered with race, class, ethnicity, and religious affiliation in analysing any given period or event, an entirely new dimension is added to social history ", Gerda LERNER, The Majority Finds Its Past, Placing Women in History, Oxford : OUP, 1979, p. 172.

3. Hasia DINER, Erin's Daughters in America: Irish Immigrant Women in the Nineteen Century, Baltimore et London : The John Hopkins University Press, 1983.

4. Janet NOLAN, Ourselves Alone: Women's Emigration from Ireland, 1885-1920, University Press of Kentucky, 1986.

5. DINER, Erin's Daughters in America, op. cit., p. 29.

6. Ibid. pp. 30-31.

7. $28 \%$ des émigrants juifs étaient des enfants, alors que les enfants ne représentaient guère que $5 \%$ des émigrants irlandais.

8. "Thus, the percentage and the absolute number of female immigration from Ireland rose in direct proportion to the hardening of family lines and the circumscribing of opportunities for women in Ireland. Women did not predominate among Irish newcomers to Britain until the 1890s, whereas among those Irish who cast their lot in Australia, men and women came in equal numbers until 1911 and then women took the lead », DINER, Erin's Daughters in America, op. cit., p. 33.

9. The Poor Law Guardians, en anglais. Membres de comités élus dan chaque paroisse, chargés de surveiller le bon fonctionnement de la workhouse, selon les termes de la Loi sur les Pauvres irlandaise de 1838.

10. Helen BURKE, The People and the Poor Law in $19^{\text {th }}$-century Ireland, Littlehampton: Women's Educational Bureau, 1987, p. 199.

11. Ann ROSSITER, « Bringing the Margins into the Centre », pp. 177-202 in Ailbhe SMYTH (ed.), Irish Women's Studies' Reader, Dublin : Attic Press, 1993.

12. "The extent of this emigration of single Irish women, travelling alone and independently of parents or husbands, was an anomaly in the history of overall European emigration to the United States ", ibid., p. 181.

13. Grace NEVILLE, «'She Never Then After That Forgot Him': Irish women and Emigration to the United States in Irish Folklore ", Mid-America: An Historical Review, Vol 74, No. 3, octobre 1992, pp. 271-289.

14. Les Archives de la Commission du Folklore Irlandais contiennent 1,5 millions de pages d'entretiens réalisés dans les années 1930 auprès de personnes âgées dans toute la campagne irlandaise. La plupart étant nées peu après la Famine, leur témoignage permet d'avoir une idée de la vie en Irlande lors de la seconde moitié du dix-neuvième siècle et le début du vingtième siècle. C'est en 1945, à l'occasion du centenaire de la Famine, qu'un questionnaire spécifique fut distribué, qui permit de recueillir des informations sur le mouvement migratoire féminin.

15. "Female emigrants, despite their greater number, flicker like shadows at the edge of perception", NEVILLE, «She Never Then After That Forgot Him », op. cit. p. 272.

16. "This remarkable compilation has not been used by any of the historians of female Irish emigration and was consulted by the present writer perhaps because most of it is in Irish », ibid.

17. " [...] a stylised repertoire of images, motifs, short narratives and supernatural legends, many of them part of a wider international narrative repertoire, which form a system of representation of famine and scarcity as well as a guide to behaviour during such crises ", Niall O'CIOSAIN, « Approaching a folklore 
archive: the Irish Folklore Commission and the Memory of the Great Famine », Folklore, Vol. 115, No. 2, août 2004, p. 225.

18. "As the memory of the Famine was transmitted, it was probably those elements of local memory that corresponded most closely to popular memory that were most "tellable" that survive in the narrative repertoire of the informants of the 1930s and 1940s ", ibid., p. 226.

19. "Girls suspected of having been pregnant were not allowed to enter the United States ", cité dans NEVILLE, «She Never Then After That Forgot Him », op. cit., p. 276.

20. "Women were one-dimension creatures who, unlike men, took the emigrant boat because of some aspect of their relationship with the opposite sex ", ibid., p. 281.

21. Thirteenth Annual Report of the Poor Law Commissioners, app. B.No. 20, H.C.1847 (816) (873) xxviii.

22. Joseph ROBINS, The Lost Children, Dublin : IPA, 1980.

23. Ibid., p. 180.

24. George STEINER, Language and Silence: Essays 1958-1966, Londres : Faber and Faber, 1985, p. 71.

25. John BANVILLE, Birchwood, Londres : Norton and Company, 1987, p. 143.

26. Margaret KELLEHER, The Feminization of Famine, Expressions of the Inexpressible?, Durham : Duke University Press, 1997.

27. "The story of Famine may be told as one of resistance or of passive victimization ", ibid., p. 5.

28. "In the majority of famine representations, the "single individual", the victim of famine who is the subject of a detailed description, is female ", ibid., p. 6.

29. "It was true : the unhappy girl had passed into another life; but whether from a broken heart, caused by sin, shame and desertion, or from famine and the pressure of general destitutiton and distress, could never be ascertained », ibid., p. 35.

30. "Because of the "sin" of her unmarried status, Margaret's vulnerabilty and victimization are of a moral rather than socio-economic nature, further obscuring details of her starvation », ibid.

\section{RÉSUMÉS}

Depuis les années 1980, l'historiographie s'intéresse aux spécificités de l'émigration féminine en Irlande. Ainsi, les femmes furent proportionnellement plus nombreuses parmi les immigrants irlandais aux Etats-Unis que dans d'autres groupes nationaux, et elles se distinguèrent par la forte proportion de célibataires. Si certaines bénéficièrent de projets d'aide à l'émigration, les conditions assorties conduisirent nombre de mères célibataires à abandonner leurs enfants. Quant aux archives de la Commission du folklore irlandais, elles permettent d'éclairer la situation de ces émigrantes, malgré le statut problématique de certains récits. Cela est lié à l'ampleur de la Grande Famine, dont l'horreur touche à l'indicible; la dimension symbolique de la femme et de la mère a ainsi pu servir à exprimer ce qui ne pouvait être représenté.

Since the 1980s historians have focused on the specificity of female Irish emigration. Women were proportionally more numerous among Irish immigrants in the United States than in other ethnic groups, and they included a higher proportion of single women. If some availed themselves of assisted emigration schemes, the conditions led many single mothers to abandon their children. The archives of the Irish Folklore Commission help to better understand the situation of Irish women emigrants, despite the problematic nature of some tales. It may be linked to the scale of the Great Famine, the horror of which was impossible to express, and the 
symbolic dimension of womanhood and motherhood may have been used to say what could not be represented.

\section{AUTEUR}

NATHALIE SEBBANE

Université Sorbonne Nouvelle 